\title{
Comparative study of Existing Models for Online Social Network
}

\author{
Vikas Attri a, and Ankush Kapoor ${ }^{\text {b }}$ \\ A \\ Assistant Professor, Chandigarh University \\ ${ }^{\text {b} A s s i s t a n t ~ P r o f e s s o r, ~ C h a n d i g a r h ~ U n i v e r s i t y ~}$
}

Article History: Received: 11 January 2021; Accepted: 27 February 2021; Published online: 5 April 2021

\begin{abstract}
Today, Online Social Networks becomes the first choice for businesses to broadcast their campaigns for branding, publicity, strategies, advertising, marketing, social influence and so many other areas. Social Network is a platform for communicating with social actors and Social Media is used by companies for broadcasting information. Online Social Network used by businesses for number of purposes but the primary concern is build new social connections that helps to target most audiences for successful campaign purposes. In OSNs sites the social objects are represented by nodes and the term edge used for connection between nodes under the graph theory. Today Social Network sites have becomes most exploded as compared to traditional sites because of impact of so many influence models over traditional models.Some of popular websites of OSN such as MySpace, Facebook, Flickr, YouTube, Google Video, Orkut, LinkedIn, Live Journal and BlogSpot have great impact on customer when targeting the sales marketing funnel for businesses. Adjacent users sometimes called engaged users tend to have more trust level as compared to random pairs users on the social media sites. Already have so much research that helps to calculate the trust factor using influence modeling. So influence models play a vital role to predict the behavior of the customer that helps to fulfill the goal of the business. The key contribution of this work is study of online social networking models.
\end{abstract}

Keywords: Online Social Network (OSN), Social Media, OSN Models, Social Networking.

\section{Introduction}

Social Influence Structure also called Social Network Analysis means considers the scenario how Facebook, Twitter algorithms try to predict products on the basis of your behavior or sometimes analyzing the additional parameters such as engagement, location, interest, reviews and so on[1][2]. Social Influence Structure means consider the deep study of the nodes, edges, protocols, social media, social network, relationship, influence parameters on basis user build the account, connect with friends, engage through social media so that target the new traffic for successful targeting the customer acquisition process[3][4].

Social network analysis helps to study how individuals distribute their behavior influence between different social groups over time [5]. Social Network analysis used to enable data segmentation such as page-views, unique page-views, average time on page, entrance rate, bounce rate, exit rate on the basis of user behavior that evaluated using analytics tools. So Social Network Analysis means computational analysis of matrices that associated with data collection methods on social media. Nodes connected in SNA either directed graph or undirected graph. In directed graph direct relationship between nodes so Twitter Social Graph is the example of directed relationship [6].

Social network categorized on the basis of strong relationship and weak relationship in which strong ones tieup are most strongly clustered as compared to weak edges relationships while weak edges tie-up known for longer-distance relationship[7][8]. In fact for lifting the viral marketing campaigns on the basis of advocacy feature of marketing funnel number of research efforts have been proposed in past studies[9][10][11].

\section{Directed vs. Undirected graphs}
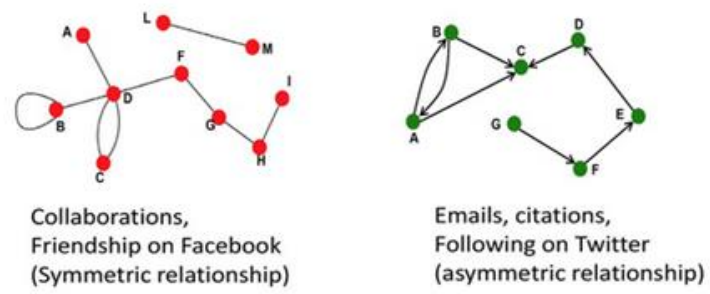

Figure 1.1. Directed vs. Undirected graphs

There is another type of relationship called undirected relationship in which consider facebook social graph is undirected relationship [12][13]. Populations in SNA represented by graph theory in which nodes and edges used for social groups. Entity or actor in the graph is called node and relationship between nodes represented by 
edges[14]. A Social Network can be defined as a Graph $G=(V, E)$, where V is the set of vertices which represents the nodes in the network and $\mathrm{E}$ is set of edges with which all the nodes in the network are connected. For the real world nodes represent the individuals in the network and edge represents the social relationship between the individuals[15][16][17].

\section{Influence models in online social networks}

Behavior analysis using advocacy approach in digital marketing sales funnel becomes challenging job for the researchers[18]. Influence models play very important role for deciding either how people can influence each other on the basis of action strategy policies they have implemented on social network sites and E-commerce sites. But implementing predictive analytics and prescriptive analytics on influence models becomes powerful strategy to increase the revenue and predict customer behavior retention policy[19][20].

Many approaches presented the influence models theory address the problem of how to measure the influence probability metric. But still very limited work on strength, limitations and advocacy phase of sales funnel in which customer have to put reviews about the service.

Author proposed the study of different influence models along with their algorithms that helps to measure social influence [21]. Authors considered the viral marketing scenario in which focused on influence maximization problem and applications of influence models [22]. Authors also discussed how to implement optimized influence maximization in social network. Our survey approach firstly examines the literature of maximum OSN models and then divides the influence models on the basis of static influence, dynamic influence, diffusion influence and behavior influence categories[23][24].

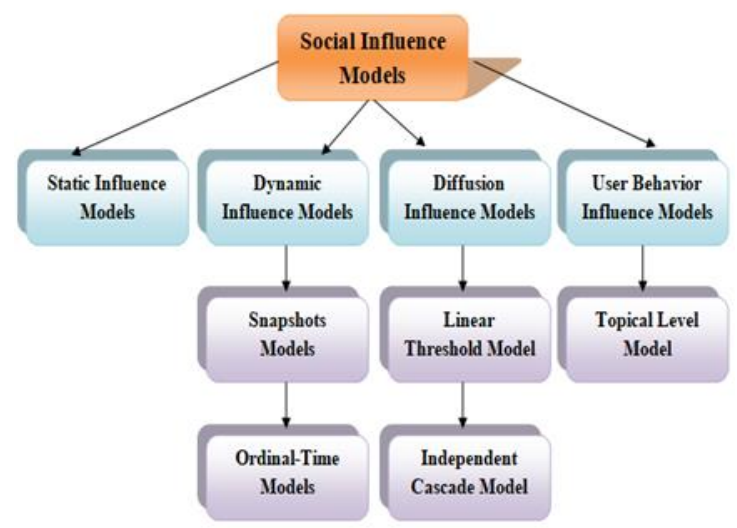

\subsection{Static Influence Models}

Figure2.1 Social Influence Models

Static Influence Models are the simplest approach having fix network size considered on OSN. In this modeling approach probability on the basis of influence parameter remains static and also independent of time factor. Bernoulli distribution is the main theory used by one instance of Static Influence Models. In Bernoulli distribution we decide what the distribution of influence on OSNs is.

\subsection{Dynamic Influence Models}

Today in digital marketing scenario companies have many campaign choices such as facebook campaign, twitter campaign, linkedin campaign, adword campaign that will dynamically affect the purchase behavior of customer. So Dynamic Influence Models have great impact on customer loyalty program. Using these campaigns user influence can change time to time. User influence and loyalty for targeting any product or join any community strongly dependent on what reviews, post and engagement activity performed by its neighbors. Here we survey two influence models of social network under dynamic influence feature. The first model is "Snapshot model" and second model is "Ordinal-time model" that based on time sequence of social network.

\section{a) Snapshot model:}

In today competition market scenario Company needs to share their brand story for growing their business followers so that people get engaged and converted into customers [41]. Cosley D et. al [42] used approach in which at two different times two Social network snapshots considered for representing the snapshot model. For the first snapshot author considered k-exposed set in which record of all individuals and ps (k) become adopters for the second snapshot at another time variation. To predict whether the behavior adopted or not, imagine all $\mathrm{k}-$ exposed nodes.

\section{b) Ordinal-time model}

This model based on time sequence of social network. Ordinal-time model creates the new link over the social network or new behavior adopted by the new individual depends on the moment-by-moment measurement parameter. The nonadopter decision either influence adopted or not depends on the basis of if nonadopter follow kth neighbor that is already adopter using the time sequence approach. Authors proposed number of neighbors in community vs. probability graph through curves on the basis of different campaign on Wikipedia. 


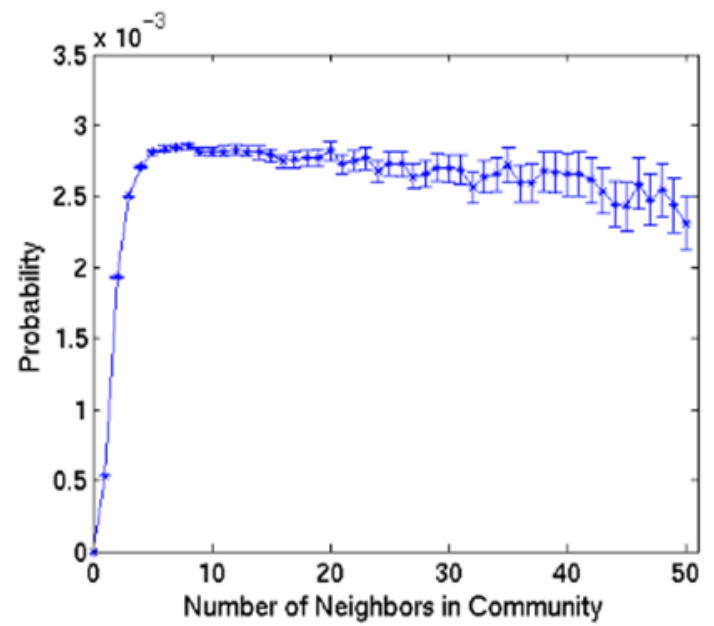

(a) Wikipedia: Ordinal-Time Definition

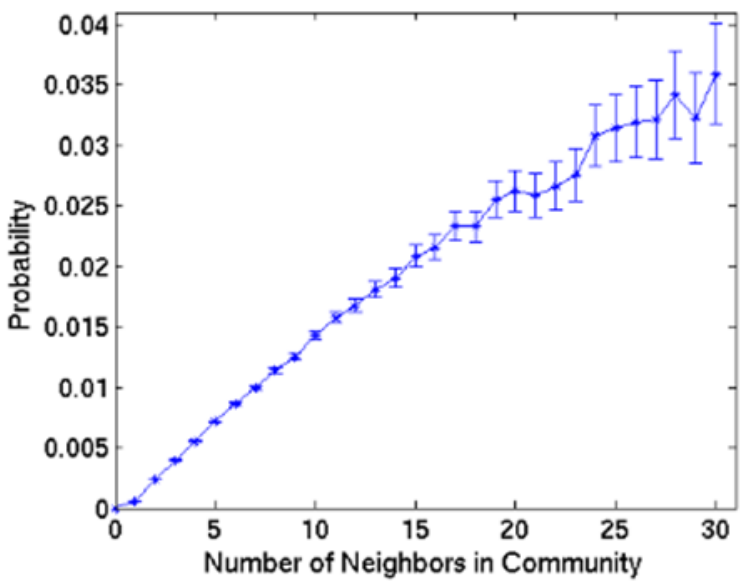

(b) Wikipedia: Snapshot Definition

\subsection{Diffusion Influence Models}

Figure 2.2 Ordinal vs. Snapshot

Diffusion as its name suggests process in which information, post and audience behavior spread over the network. For example new product influence after completing brand awareness campaign only target the small scale audience in which limited few "early adopters". As more audience adopted the product obviously influence on their friends and neighbors so that creates a cascade. Sometimes diffusion influence models also create the influence maximization problem due to fake posts, fake reviews and fake engagement [30].

Authors [30] discussed the influence maximization problem and also proposed a framework that tries to sort influence maximization problem. The framework based on probabilistic modeling of interaction in which empirical study conducted in the context of viral marketing that considers nodes as customers.

\section{a) Linear threshold model}

This approach facilitates each user in the network carry a threshold. User adoption of product depends on if other user in the group already adopted the product. Users with higher threshold don't need to worry about when adopt a product and user having lower threshold will need to tend either to follow someone or not. Author discussed fundamental algorithm problems related to viral marketing strategies and also proposed algorithms that based on natural greedy strategies that become $63 \%$ of optimal for businesses decisions. Authors also discussed how the optimized solution based on degree centrality and distance centrality notions of social analysis [30].

\section{b) Independent Cascade Model}

As we discussed in linear threshold model decision influence depends upon the propagation of like information or viral news infection so situations when the node have chance to get infected because in contact with an infected node. News rumors also one of the current scenarios of linear threshold model. But in independent cascade model consider we have some network and initial set of active nodes. In this model firstly focus on two first customer on social media those already bought some product. The rule is on every step an active node $u$ can activate the connected neighbor $\mathrm{v}$ with a probability pu, $\mathrm{v}$ (single chance) and in this probability model the probability depends on the edge but the key here that every node activate its neighbor through a single chance only. 


\subsection{User Behavior Influence Models}

Author [30] discussed parameters that helps to distinguish social influence on the basis of topic as well also try to quantify strength of model by proposing new model. Authors proposed the Topical Affinity Propagation (TAP) that designed with efficient distributed learning algorithms under Map-Reduce framework. Author also discussed the problem of maximization and after try to solve the problem using Expectation Maximization (EM) algorithm. Author considered the independent cascaded model for implementing the proposed algorithm. Using Expectation Maximization (EM) algorithm the influence probability also increased in each iteration.

\section{Comparative analysis of OSN models}

In recent years, OSN sites have become an emerging mainstream medium, creating a dramatic social and economic impact in the world. OSNs play a vital role in delivering resources for a number of purposes, with three traditional main positions among other locations. Second, the OSNs are used to maintain and improve current social ties and to construct a new social environment across the globe. Second, OSNs are abused to share their knowledge with their social neighbors, either privately or publicly, as professionals, and to express their opinions in public. Third, it is used to classify novel and interesting content via the filtering and recommendation process.

Many scholars are focusing on the discovery and resolution of various dimensions of social networks. In order to address the issues, the available literature is updated in order to locate differences between the current bodies of information. It is important to compile the information gathered in the past. The study of the social network allows researchers to discover the mechanisms used in social systems and to find the pattern of interaction between individuals that have emerged from research topics such as sociology, epidemiology, the recommendation framework, and criminology.

Khan and Lee [24] suggested a new paradigm of evolution of scale-free social networks (SNs) depend on homophily merged with preferential extensions. A generic SN synthesized dataset containing SN nodal elements, like demographic elements, spatial data or consumer profits, is obtained. Homophilia is one of the main factors in the development of SN interactive relationships. User elements and similarities are used to integrate SN maps. Li et al. [25] provided a systematic study of knowledge dissemination studies in SN. It splits the models of data diffusion into 2 classifications: explaining and predictive designs.

The idea of a DA to calculate the impact of online social network actors such as Twitter was proposed by Goritz et al. [26]. The article compares the influence of the IPA with that of other artists by implementing ERGMs depend on Twitter info in weather modify negotiations. The results show that IPAs have the same authority as state artists in Twitter's world weather climate $\mathrm{CN}$.

Zhang et al. [27] have introduced a novel 2-stage gossip distribution or rebuttal model with a time effect for OSNs. The dynamic mechanism for the propagation and refutation of rumors with a time impact is thoroughly examined. This is followed by a 2-stage design and related mean-area formulas in both homogeneous and heterogeneous systems. The Monte Carlo simulations are performed to explain the dynamics of gossip transmission or rebuttal in both the WSN and the BAN.

Alnajran et al. [28] proposed a NHDP approach to improving the efficacy of SM in the Twitter tweet context. The components of the suggested pre-processing technique are debated or analyzed as part of SemEval-2014's joint job in an annotated data collection. A test with the cosine angle was performed to assess the effect of our approach on the base line (C-Method). The results show that the system has correlations and error rates above the baseline.

Hufbauer et al. [29] proposed a design for creating SN that nearly comparison real network OSN devices (e.g. Facebook and Twitter) including group frameworks. The model is scalable, stable and novel. An automatic variable tuner that could meet the performance of the design to an OSN geology is also proposed. The design could then be utilized as a data factory to create integrated test beds for topology. The model is calibrated to fit 2 huge real-world OSN systems tests, the BAM and the LFR standard utilized as a baseline.

Tang et al. [30] proposed hop-based methods that could be simply scaled to millions of nodes and billions of boundaries. Xia et al. [25] suggested the SNURank algorithm, a mining algorithm for opinion leaders in the social network. It can be used to measure the power of the user and to pick opinion leaders by defining the degree of attention depend on the customer's attention behavior analysis or the ideas of the PageRank algorithm.

Ullah and Lee [31] have suggested the implicit group detection clustering method, based on a trust modeling that weighs the interest of users in a specific OSN. Trust modeling is measured by the user's benefit in grouping users into separate categories of better rates and authenticity resemblances within clusters. The structure plan clustering method operates by listing the nodes by the weighted degree instead and choosing the original community centers which are not in another's neighbors. The deterministic trust platform is developed to forecast the sense of faith among customers and superiors.

A research on the effect of EC on tax and accounting practice was released by Branda et al. [32]. The goal of this investigation is to research the effect of tax and e-commerce accounting operations in the context of the faster growth of Internet transactions. The need for this study is dictated by the current context in which social networks are increasingly being used as forums for the promotion of electronic business. The paper also aims to highlight the need to develop an international system of income/profit taxation as it does not currently exist. To endorse this 
strategy, two scenarios of electronic and conventional trading in which e-commerce prospects are illustrated and the major problem of identifying the source of income is demonstrated.

Cao et al. [33] have developed a multi-stage rural e-commerce reverse logistics network platform under the B2C model, taking into account both cost savings and customer satisfaction. The GA 2-phase heuristic nesting method is applied to validate the feasibility of a method with a research study of $3 \mathrm{C}$ particles in Anhui Province. The findings demonstrate that the user satisfaction of the scheme in the nearby region is maximized and that the scheme has capability, efficiency, large quality and high risk owing to the low of the extra factor of the scheme and the standardization of resource input.

Liu et al. [34] proposed a credit risk gambling mechanism depend on game postulate and rational net concept of Petri. Second, a theoretical simulation solution known the LGPN is suggested by incorporating the Logical Petri nets into the game theory. The utility functions that explain the relationship among clients in various marketing strategies are retrieved in order to define the benefits that each company acquired all through competition procedures using the least square approach; the next is the Petri Net Logical Game Method for evaluating the interplay among different organizations in the Enterprise Competition Subsystem. An reachable marking graph for Logical Game Petri net system is introduced for behaviour analysis. Findings outcomes present that when companies selects a BM of true administration with a likelihood of 58.95 per cent or more, firms will achieve relatively stable and high expected value.

Liu et al. [35] aimed to resolve the last-mile distribution of RECL for the survival of a third-party logistics business. Evaluating the effectiveness of the RECL (longer travel chain and low consumption volume), the path optimal solution is created to achieve the last mile of RECL in addition to increasing the income of the governmentfunded logistics business. To fix the method, the ACO was enhanced to fit the last mile distribution of RECL by altering the heuristic specifics, the pheromone upgrade legislation, and the answer installation. The outcomes demonstrated that the proposed ACO could offer a feasible route plan for the last mile of arrival of the RECL.

Wu et al. [36] addressed the implementation of $\mathrm{C} 2 \mathrm{~B}$ e-commerce in three areas: $\mathrm{C} 2 \mathrm{~B}$ connotation and definition confusion, $\mathrm{C} 2 \mathrm{~B}$ network and application bottleneck, and $\mathrm{C} 2 \mathrm{~B}$ application strategy and prospect. While $\mathrm{C} 2 \mathrm{~B}$ technology is still in its infancy, consumer-driven e-commerce must be the future of e-commerce. The author claims that more third-party e-commerce sites would be moved from B2C applications to C2B applications.

George et al. [37] examines the context of the customer's objective to buy goods from the C2C EC policy by evaluating 2 mediating parameters: consumer confidence in traders and user trust in suppliers of C2C EC platforms. The survey is made up of 435 respondents from consumers of e-commerce in Indonesia. Results have shown that the credibility of sellers, the quality of information and the quality of service offered by sellers have a positive effect on customers' trust in vendors. Meanwhile, IQ, SQ or policy credibility have a positive impact on the confidence of the users in the $\mathrm{C} 2 \mathrm{C}$ e-commerce platform.

Yang et al. [38] developed and introduced BPGS by smart contracts. The blockchain-based PGS (BPGS) for this business model was suggested. Big data. The blockchain is a decentralized framework that can accelerate product checks verification. Moreover, for the BPGS suggested, 51\% of attacks cannot be reached without simultaneously compromise between $51 \%$ of the traders and e-commerce firms.

In addition to the cost assessment model, Joshi et al [39] focused on different concerns issues linked to previous $\mathrm{EC}$ for $\mathrm{C} 2 \mathrm{C}$. Using blockchain in this situation helps to move the model to a distributed one, so that customers can share better without exchanging personal information and products at the right price. The intention was to improve the price assessment of utilize goods, to offer end-users with information security or trust by utilizing various aspects of block chain technology.

Jude et al. [40] examined the e-readiness of small to medium-sized enterprises (SMEs) in Windhoek, Namibia, by introducing an e-Commerce Business to Consumer (B2C) business model as part of their marketing strategy. As SMEs aim an significant part in the NE, their participation in e-commerce is crucial. Quantitative data was obtained by the use of survey questions to determine their e-readiness and analyzed using the Social Sciences Statistical Kit (SPSS). Findings have shown that small and medium-sized businesses in Namibia are able to embrace B2C e-commerce.

Table 1. Comparative Analysis of OSN Models

\begin{tabular}{|c|c|c|c|}
\hline Year & Title & Model & \multicolumn{1}{|c|}{ Remarks } \\
\hline 2018 & $\begin{array}{c}\text { OSN Evolution Model Based on } \\
\text { Homophily and Preferential Attachment. } \\
\text { Model }\end{array}$ & SN & $\begin{array}{l}\text { Graph shows a } \\
\text { similar description of } \\
\text { real-life SN maps with } \\
\text { user elements. }\end{array}$ \\
\hline
\end{tabular}




\begin{tabular}{|c|c|c|c|}
\hline 2020 & $\begin{array}{l}\text { International Public Administrations on } \\
\text { Twitter: A Comparison of Digital Authority } \\
\text { in GCP. }\end{array}$ & $\begin{array}{l}\text { Exponential } \\
\text { Random Graph } \\
\text { Model (ERGM) }\end{array}$ & $\begin{array}{l}\text { IPA is } \\
\text { allocated as much } \\
\text { program me as the state } \\
\text { artists on Twitter in } \\
\text { world weather CN. }\end{array}$ \\
\hline 2018 & $\begin{array}{l}\text { Modeling rumor propagation and } \\
\text { refutation with time effect in OSN. }\end{array}$ & $\begin{array}{c}\text { Watts-Strogatz } \\
\text { and Barabási-Albert }\end{array}$ & $\begin{array}{l}\text { Identifying rumor } \\
\text { has improved } \\
\text { specifically in order to } \\
\text { reduce the negative } \\
\text { effects of rumor } \\
\text { effectively. }\end{array}$ \\
\hline 2018 & 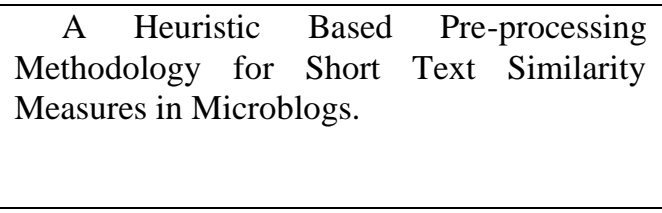 & $\begin{array}{l}\text { Heuristic } \\
\text { Modelling }\end{array}$ & $\begin{array}{l}\text { Cosine angle is used } \\
\text { to evaluate the effect on } \\
\text { the baseline (C-Method), } \\
\text { in means of correlations } \\
\text { or error levels. }\end{array}$ \\
\hline 2020 & $\begin{array}{l}\text { A Proximity-Based Generative Model for } \\
\text { Online Social Network Topologies. }\end{array}$ & $\begin{array}{l}\text { Barabasi-Albert } \\
\text { and Lancichinetti- } \\
\text { Fortunato-'Radicchi }\end{array}$ & $\begin{array}{l}\text { This model can be } \\
\text { used to generate datasets } \\
\text { for training or research. }\end{array}$ \\
\hline 2017 & $\begin{array}{l}\text { Influence Maximization Meets Efficiency } \\
\text { and Effectiveness: A Hop-Based Method. }\end{array}$ & $\begin{array}{c}\text { Infleunce } \\
\text { Maximization }\end{array}$ & $\begin{array}{l}\text { The OSN datasets } \\
\text { show productivity and } \\
\text { effectiveness. }\end{array}$ \\
\hline 2016 & $\begin{array}{l}\text { Mining Algorithm of Opinion Leaders } \\
\text { Based on Analysis of User Attention } \\
\text { Behavior. }\end{array}$ & $\begin{array}{l}\text { Page Rank } \\
\text { Algorithm, SNURank } \\
\text { algorithm }\end{array}$ & $\begin{array}{l}\text { The influence value } \\
\text { of user can be } \\
\text { determined by the degree } \\
\text { of attention. It is more } \\
\text { reliable to identify the } \\
\text { opinion leaders in social } \\
\text { networks. }\end{array}$ \\
\hline 2017 & $\begin{array}{l}\text { Community clustering based on trust } \\
\text { modeling weighted by user interests in OSN. }\end{array}$ & $\begin{array}{l}\text { Probabilistic } \\
\text { Modeling }\end{array}$ & $\begin{array}{l}\text { Community } \\
\text { Clustering Approach } \\
\text { evaluates the framework } \\
\text { effectively by having a } \\
\text { weighted subgraph for } \\
\text { the community and less } \\
\text { for the another groups. }\end{array}$ \\
\hline
\end{tabular}

\section{Summary}

OSN is the most popular virtual community, allowing users to discover, enlarge, and exploit their network. Many social network users share their information and opinions through a variety of OSN sites, like Facebook, Twitter, MySpace, or LinkedIn. Social Network has the potential to link diverse individuals and communities globally. The increasing scale of the social network makes it easier for social users to take the perceived ideas to their advantage. A comparative study of various OSN and E-Commerce models has been presented in this paper. From the literature survey it is inferred that the issue in social networking like protection, privacy, etc. can be solved by using different techniques such as diffusion modeling, probabilistic modelling, etc. There are several potential future directions for expanding these models of social control to overcome perceived drawbacks such as scalability and performance. A combination of these models can produce more accurate results and help to overcome individual limitations.

\section{References}

1. Aggrawal, N., Ahluwalia, A., Khurana, P., Arora, A., 2017'Brand analysis framework for online marketing: ranking web pages and analyzing popularity of brands on social media" Soc. Network Analy. Mining 7 (1), 21.

2. AlAwadhi, S., Parycek, P., \& Kesan, J. P., "Introduction to Social Media and Social Networking Minitrack," 2013 46th Hawaii International Conference on System Sciences, pp. 1992, 2013.

3. Ali, S., Islam, N., Rauf, A., Din, I., Guizani, M., \& Rodrigues, J., "Privacy and Security Issues in Online Social Networks," Future Internet, vol. 10, no. 12, pp. 114, 2018.

4. Alnajran, N., Crockett, K., McLean, D., \& Latham, A., "A Heuristic Based Pre-processing Methodology for Short Text Similarity Measures in Microblogs," 2018 IEEE 20th International Conference on High Performance Computing and Communications; IEEE 16th International Conference on Smart City; IEEE 4th International Conference on Data Science and Systems (HPCC/SmartCity/DSS), 2018. 
5. Alrubaian, M., Al-Qurishi, M., Almri, A., Al-Rakhami, M., Hassan, M. M., \& Fortino, G., "Credibility in Online Social Networks: A Survey," IEEE Access, vol. 7, pp. 2828-2855, 2018.

6. Arnaboldi, V., Passarella, A., Conti, M., \& Dunbar, R. I. M. (2015). Conclusion. Online Social Networks, 93-100.

7. Branda, C., Megan, O., \& Craciunescu, M., "Study on the Impact of E-Commerce on Tax and Accounting Activities, World Applied Sciences Journal, vol. 24, no. 4, pp. 534-539, 2013.

8. Cao, F., Guo, J., \& Liang, C., "Multi-stage Layout Optimization for Reverse Logistics Network of Rural E-Commerce under B2C Mode: A Case Study of E-Commerce Enterprises on 3C Products," 2016 IEEE 13th International Conference on e-Business Engineering (ICEBE), 2016.

9. Doloto, U., \& Chen-Burger, Y., “A Survey of Business Models in eCommerce,” Jan. 2015.

10. Erdos, P., Renyi, A. (1960). "On the evolution of random graphs", Publications of the Mathematical Institute of the Hungarian Academy of science 5.

11. Fire, M., Goldschmidt, R., \& Elovici, Y., "Online Social Networks: Threats and Solutions," IEEE Communications Surveys \& Tutorials, vol. 16, no. 4, pp. 2019-2036, 2014.

12. Fuciu, M., \& Gorski, H., "Marketing Research regarding the Usage of Online Social Networking Sites by High School Students," Procedia Economics and Finance 6, pp. 482-490, 2013.

13. George, V., Pono, L. R., \& Sandhyaduhita, P. I. "Trust in Merchants and E-commerce Platforms as Antecedents of Users' Purchase Intention in C2C E-Commerce,” IEEE Xplore, Sep. 2020.

14. Goritz, A., Schuster, J., Jörgens, H., \& Kolleck, N., "International Public Administrations on Twitter: A Comparison of Digital Authority in Global Climate Policy," Journal of Comparative Policy Analysis: Research and Practice, pp. 1-25, 2020.

15. Hufbauer, E., Hudson, N., \& Khamfroush, H., “A Proximity-Based Generative Model for Online Social Network Topologies," 2020 International Conference on Computing, Networking and Communications (ICNC): Social Computing and Semantic Data Mining, pp. 648-653, 2020.

16. Joshi, P., \& Kumar, A., "A Novel Framework for Decentralized C2C E-commerce using Smart Contract," 2020 11th International Conference on Computing, Communication and Networking Technologies (ICCCNT), 2020.

17. Jude O., \& Nkululeko, M., "E-readiness of Namibian SME retailers to Adopt B2C e-Commerce," ISTAfrica Conference (IST-Africa), Jul. 2020.

18. Kapoor, K. K., Tamilmani, K., Rana, N. P., Patil, P., Dwivedi, Y. K., \& Nerur, S., “Advances in Social Media Research: Past, Present and Future,” Information Systems Frontiers, vol. 20, no. 3, pp. 531-558, 2017.

19. Khan, A. G., "Electronic Commerce: A Study on Benefits and Challenges in an Emerging Economy," Global Journal of Management and Business Research: B Economics and Commerce, vol. 16, no. 1, 2016.

20. Khan, J., \& Lee, S., "Online Social Networks (OSN) Evolution Model Based on Homophily and Preferential Attachment," Symmetry, vol. 10, no. 11, 654, 2018.

21. Kim, J., \& Hastak, M., "Social network analysis: Characteristics of online social networks after a disaster," International Journal of Information Management, vol. 38, no. 1, pp. 86-96, Feb. 2018.

22. Li, M., Wang, X., Gao, K., \& Zhang, S., "A Survey on Information Diffusion in Online Social Networks: Models and Methods," Information, vol. 8, no. 4, Aug. 2017.

23. Li, Y., Cao, H., \& Zhang, Y., "Static and Dynamic Structure Characteristics of a Trust Network and Formation of User Trust in an Online Society," Social Networking, vol. 7, no. 4, pp. 197-219, Oct. 2018.

24. Liu, W., "Route Optimization for Last-Mile Distribution of Rural E-Commerce Logistics Based on Ant Colony Optimization," IEEE Access, vol. 8, pp. 12179-12187, 2020.

25. Liu, W., Feng, X., Zhang, F., Du, Y., \& Yan, C., "Analytic of B2C E - Commerce Credit Mechanism Mixed Strategy Risk Behavior Based on Logical Game Petri Nets," IEEE Access, vol. 6, pp. 29109-29131, 2018.

26. Luo, L., \& Fang, J. (2014). A Study of How the Watts-Strogatz Model Relates to an Economic System's Utility. Mathematical Problems in Engineering, 2014, 1-7.

27. Persia, F., \& D'Auria, D., "A Survey of Online Social Networks: Challenges and Opportunities," 2017 IEEE International Conference on Information Reuse and Integration (IRI), pp. 614-620, 2017.

28. Rani, S., \& Sharma, R. K., "Attitude of Accounting Professionals towards Impact of E-Commerce in Relation to their Qualification," IMPACT: International Journal of Research in Business Management (IMPACT: IJRBM), vol. 2, no. 3, pp. 127-132, Mar. 2014.

29. Shahjee, R., "The Impact of Electronic Commerce on Business Organization," Scholarly Research Journal for Interdisciplinary Studies, vol. 4, no. 27, pp. 3130-3140, 2016.

30. Singh, S. S., Singh, K., Kumar, A., Shakya, H. K., \& Biswas, B., "A Survey on Information Diffusion Models in Social Networks," Advanced Informatics for Computing Research, pp. 426-439, 2018.

31. Soryani, M., \& Minaei, B., "Social Networks Research Aspects: A Vast and Fast Survey Focused on the Issue of Privacy in Social Network Sites," IJCSI International Journal of Computer Science Issues, vol. 8, no. 6, pp. 363-373, Nov. 2011. 
32. Spiro, E., \& Ahn, Y.-Y. (Eds.). (2016). Social Informatics. Lecture Notes in Computer Science.

33. Steininger, D. M., Holz, J., \& Veit, D., "Comparing Traditional and Electronic Business Models of the Music Industry: A Content Analytical Approach," Conference: Proceedings of ConLife Academic Conference 2012, Jan. 2012.

34. Tang, J., Tang, X., Yuan, J., "Influence Maximization Meets Efficiency and Effectiveness: A Hop-Based Approach," Social and Information Networks, Aug. 2017.

35. Ullah, F., \& Lee, S., "Community clustering based on trust modeling weighted by user interests in online social networks," Chaos, Solitons \& Fractals, vol. 103, pp. 194-204, 2017.

36. Verma, S., \& Mehrotra, R., "Research Paper on Role of Analytics in Renovating Human Resource Management," IPASJ International Journal of Management (IIJM), vol. 5, no. 5, pp. 17-23, May 2017.

37. Wei, Z., Yanqing, Y., Hanlin, T., Qiwei, D., \& Taowei, L. (2013). Information Diffusion Model Based on Social Network. Proceedings of the 2012 International Conference of Modern Computer Science and Applications, 145-150.

38. Wu, Q., Ma, J., \& Wu, Z., "Consumer-Driven E-Commerce: A Study on C2B Applications," 2020 International Conference on E-Commerce and Internet Technology (ECIT), 2020.

39. Xia, Y., Cao, M., Zhang, X., \& Bae, H. Y., "Mining Algorithm of Opinion Leaders Based on Analysis of User Attention Behavior," IJISET - International Journal of Innovative Science, Engineering \& Technology, vol. 3, no. 2, pp. 357-361, Feb. 2016.

40. Yang, C.-N., Chen, Y.-C., Chen, S.-Y., \& Wu, S.-Y., "A Reliable E-commerce Business Model Using Blockchain Based Product Grading System," 2019 IEEE 4th International Conference on Big Data Analytics (ICBDA), 2019.

41. Youssef, B. E., "Online Social Network Internetworking Analysis," International Journal of NextGeneration Networks (IJNGN), vol. 6, no. 2, pp. 1-15, Jun. 2014.

42. Zhang, Y., Su, Y., Weigang, L., \& Liu, H., "Modeling rumor propagation and refutation with time effect in online social networks," International Journal of Modern Physics C, 1850068, vol. 29, no. 8, 2018.

43. Zhao, S., Yu, L., \& Cheng, B. (2017). Probabilistic Community Using Link and Content for Social Networks. IEEE Access, 5, 27189-27202. 